\title{
Calculation of Distributed Magnetic Flux Density under the Stator-Turn Fault Condition
}

\author{
Kyung-Tae Kim", Jin Hur*, and Byeong-Woo Kim ${ }^{\dagger}$ \\ ${ }^{*}$ Dept. of Electrical Eng., Ulsan University, Ulsan, Korea
}

\begin{abstract}
This paper proposed an analytical model for the distributed magnetic field analysis of interior permanent magnet-type blush-less direct current motors under the stator-turn fault condition using the winding function theory. Stator-turn faults cause significant changes in electric and magnetic characteristic. Therefore, many studies on stator-turn faults have been performed by simulation of the finite element method because of its non-linear characteristic. However, this is difficult to apply to on-line fault detection systems because the processing time of the finite element method is very long. Fault-tolerant control systems require diagnostic methods that have simple processing systems and can produce accurate information. Thus analytical modeling of a stator-turn fault has been performed using the winding function theory, and the distributed magnetic characteristics have been analyzed under the fault condition. The proposed analytical model was verified using the finite element method.
\end{abstract}

Key words: Magnetic field analysis, Stator-turn fault, Winding function theory

\section{INTRODUCTION}

Traditionally owing to their excellent performance characteristics, including a high power density and high efficiency, IPM-type blush-less direct current (BLDC) motors have been actively developed for many different kinds of the electric actuating systems in a wide variety of industrial and transit applications such as $\mathrm{HEV}, \mathrm{EV}$ and their motorized systems [1]. In particular, they have been used in many safety-critical application systems. Therefore, many methods have been proposed for fault prediction and detection in BLDC motors. When a motor develops a short circuit between the windings of one of its coils, known as a stator turn fault (STF), heat is generated by the circulating current in the shorted coil. The circulating current is induced by the magnetic linkage flux originating from the permanent magnet (PM) in the shorted turn which results in deterioration of the torque characteristic by the distortion of the air-gap magnetic flux distribution. Moreover, this heat, which increases proportionally to the square of the circulating current, expands the short-state to the entire fault phase in a very short period of time unless the fault is detected early and pre-emptive action is taken [2], [3].

Manuscript received Jan. 29, 2013; revised Apr. 23, 2013

Recommended for publication by Associate Editor Dong-Hee Lee.

${ }^{\dagger}$ Corresponding Author: bywokim@ulsan.ac.kr

Tel: +82-052-259-1287, Ulsan University

'Dept. of Electrical Eng., Ulsan University, Korea
Therefore, the analysis of motors with a STF that is reasonably accurate with respect to the magnetic nonlinearity is strongly needed. The inductance of the stator changes the operating conditions due to magnetic saturation and cross-coupled magnetization effects. For this reason, many of the studies on STFs have used the finite element method (FEM) simulation [4]-[9]. However, it is difficult to apply FEM simulation to online fault diagnosis systems because the processing time of the FEM is very long. For this reason, this paper performs STF analysis using the machine equation and proposes an analytical modeling using the winding function theory (WFT). The proposed model can calculate the stator inductance and the distributed magnetic characteristics of a system under the fault state. The proposed analytical model, which is a combination of the WFT-model of IPM type motors having a STF with a BLDC drive, is used for analyzing the distributed magnetic flux density under the STF conditions. The proposed analytical model is verified by the FEM and experimental results.

\section{STATOR-TURN FAULT MODELING}

\section{A. Stator-Turn Fault}

For the STF it is assumed that each phase winding consists of turns which are connected in series and that the three-phase concentrated windings are wye-connected with a floating neutral point. A circuit diagram of the three-phase windings with a STF in the $A$-phase winding is shown in Fig. 1-(a), 


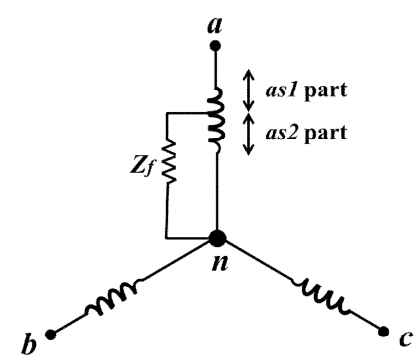

(a) Winding placement of $A$-phase.

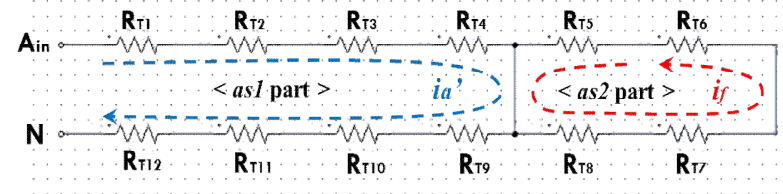

(b) Equivalent circuit in faulty phase.

Fig. 1. Schematic circuit of STF.

where as 1 and as 2 represent the healthy and the shorted turns, respectively, and $i_{f}$ represents the circulating current in the shorted turns. In addition, Fig. 1-(b) shows the equivalent circuit in the faulty phase. The input current is changed by reducing the number of turns in the as 1 section, and a circulating current is generated by the rotating magnetic field of the PM in the as 2 section.

Under the above assumptions, the stator line-neutral voltages and the developed torque of IPM type motors with an stator-turn fault on the $A$-phase winding can be represented by the $a b c$-variables in (1) [2], [4].

In addition, the "fault fraction" $\mu$, is defined as the ratio of the number of shorted turns to the number of turns per phase, and $Z_{f}$ represents the possible external impedance between the shorted turns. The voltage at the winding of an IPM type motor with a turn fault on the $A$-phase winding is represented by (2), where, $\lambda_{a}$ is the linkage flux by the phase coil current, $\lambda_{a r}$ is the linkage flux by the PM, and $L_{a m}=L_{a a}-L_{l s}$. In addition, the voltages at as 1 and as 2 can be obtained using (3) and (4), respectively. In (3) and (4), $\lambda_{a}{ }^{\prime}=L_{a m}{ }^{\prime} \cdot$ if is the linkage flux used for the consideration of the effect of the turn count as reduced by the shorted turn. When a stator-turn fault is involved with a small number of $\boldsymbol{\mu}$, the resultant asymmetry of the stator voltage generally has a small effect on the overall stator voltage. Thus (4) can be rewritten as (5). $v_{a n}$ ' represents the value of the $A$-phase-neutral voltage in the faulty state.

$$
\begin{array}{r}
v_{s n}^{\prime}=R_{s}^{\prime} \cdot i_{s}^{\prime}+L_{s}^{\prime} \cdot \frac{d i_{s}^{\prime}}{d t}+w\left(\frac{d L_{s}^{\prime}}{d \theta} \cdot i_{s}^{\prime}+\frac{d \lambda_{s r}^{\prime}}{d \theta}\right) \\
v_{a n}^{\prime}=v_{a s 1}+v_{a s 2} \\
=\left(R_{s} \cdot i_{a}+\frac{d \lambda_{a}}{d t}+w \frac{d \lambda_{a r}\left(\theta_{r}\right)}{d \theta_{r}}\right)-\left(\mu \cdot R_{S} \cdot i_{f}+\mu \cdot w \frac{d \lambda_{a r}\left(\theta_{r}\right)}{d \theta_{r}}\right)
\end{array}
$$

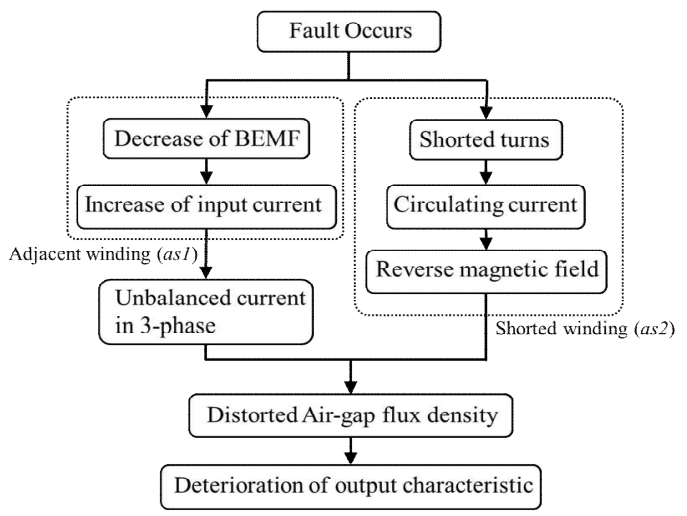

Fig. 2. Influence of stator turn fault.

$$
\begin{aligned}
v_{a s l}= & (1-\mu)\left[R_{s} i_{a}+L_{a}\left(\theta_{r}\right) \frac{d i_{s}}{d t}+w \frac{d L_{a}\left(\theta_{r}\right)}{d \theta_{r}} i_{s}+w \frac{d \lambda_{a r}\left(\theta_{r}\right)}{d \theta_{r}}\right], \\
& -\mu(1-\mu)\left[L_{a m}\left(\theta_{r}\right) \frac{d i_{f}}{d t}+w \frac{d L_{a m}\left(\theta_{r}\right)}{d \theta_{r}} i_{f}\right] \\
v_{a s 2} & =\mu\left[R_{s} i_{a}+L_{a}\left(\theta_{r}\right) \frac{d i_{s}}{d t}+w \frac{d L_{a}\left(\theta_{r}\right)}{d \theta_{r}} i_{s}+w \frac{d \lambda_{a r}\left(\theta_{r}\right)}{d \theta_{r}}\right], \\
& -\mu\left[R_{s} i_{f}+L_{l s} \frac{d i_{f}}{d t}+\mu\left(L_{a m}\left(\theta_{r}\right) \frac{d i_{f}}{d t}+w \frac{d L_{a m}\left(\theta_{r}\right)}{d \theta_{r}} i_{f}\right)\right] \\
v_{a n}^{\prime}= & \frac{R_{f}}{\mu} i_{f}+\left\{R_{s} \cdot i_{f}+\left[L_{l s}+\mu \cdot L_{a m}\right] \frac{d i_{f}}{d t}+\mu \cdot w \cdot \frac{d L_{a m}}{d \theta_{r}} i_{f}\right\}
\end{aligned}
$$

where $v_{s n}^{\prime}=\left[\begin{array}{llll}v_{a s 1} & v_{a s 2} & v_{b n} & v_{c n}\end{array}\right]^{\mathrm{T}} ; R_{s}^{\prime}=\operatorname{diag}\left[(1-\mu) R_{s} \mu R_{s} R_{s} R_{s}\right]$; $\lambda^{\prime}{ }_{s r}=\left[(1-\mu) \lambda_{a r} \mu \lambda_{a r} \lambda_{b r} \lambda_{c r}\right]^{\mathrm{T}} ; i_{s}^{\prime}=\left[\begin{array}{ll}i_{a} & i_{a}-i_{f} i_{b} i_{c}\end{array}\right]^{\mathrm{T}}$.

\section{B. STF Modeling Using Winding Function Theory}

Influence of a STF can be described by Fig.2. Finally, the cause of the deterioration of the output characteristics is the distorted air-gap flux density. Therefore, the fault analysis requires magnetic distributed characteristics under the STF condition. To analyze the magnetic distributed characteristic under the STF condition, various magnetizations and mutual inductances of the motor must be calculated.

In this paper, the WFT was used for the analysis of STFs because WFT is a useful technique for modeling the magneto motive force which occurs in the stator windings [3].

The turn function (TF; function of the conductor current density) and the winding function (WF; function of the winding distribution in the stator) can be described as in Fig. 3 and calculated as in (6). The value of these functions depends on geometry because it takes the number of turns and poles per phase into account. $\mathbf{n}_{\mathbf{A}}$ and $\mathbf{N}_{\mathbf{A}}$ represent TF and WF. As shown in (7), the value of TF and WF can be rewritten as $t_{-} h$ and $T \_h$.

$$
\begin{gathered}
t \_h=\frac{\text { Total number of turns }}{\text { Number of slots per phase }}=\frac{N}{3}, \\
T_{-} h=\frac{t_{-} h}{2}=\frac{N}{6}
\end{gathered}
$$

Based on these functions, the self-inductance $\left(L_{x x}\right)$ and 


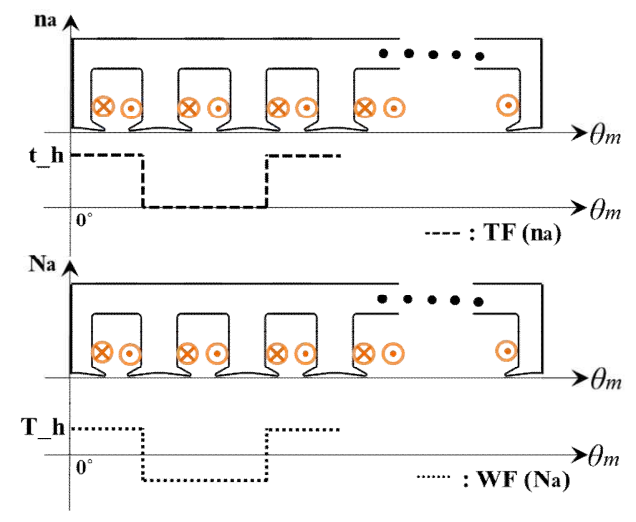

Fig. 3. TF and WF of $A$-phase under steady state.

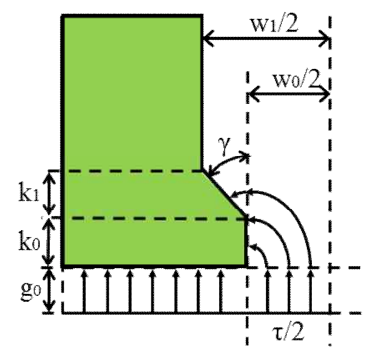

Fig. 4. Flux line distribution due to the stator slots.

mutual-inductance $\left(L_{x y}\right)$ can be calculated and used in the voltage equation as shown in (7). Equation (8) is the voltage equation of IPM type motors.

$L_{x x}=\mu_{0} \cdot r \cdot l_{s t} \cdot\left[\int_{0}^{2 \pi} g^{-1}\left(\theta_{m}-\varphi\right) n_{x}\left(\theta_{m}\right) N_{y}\left(\theta_{m}\right) d \theta_{m}\right]$

$L_{x y}=\mu_{0} \cdot r \cdot l_{s t} \cdot\left[\int_{0}^{2 \pi} g^{-1}\left(\theta_{m}-\varphi\right) n_{x}\left(\theta_{m}\right) N_{y}\left(\theta_{m}\right) d \theta_{m}\right]$

where, $x$ is the $A$-phase of the TF; $y$ is the $A$-phase of the WF; $r$ is the radius of the rotor; $l_{s t}$ is the stack length; $\varphi$ is the rotating angle; and $g^{-1}\left(\theta_{m}\right)$ is the inverse air-gap function of an IPM motor.

$$
V_{s}=R i+L \frac{d i}{d t}+i \frac{d L}{d t}+e
$$

In the case of IPM motors, the magnetic resistance varies according to the rotor position. This variance is due to the position of the buried PMs, and it is called the magnetic saliency. Thus in order to compute the air-gap flux density, it has to be considered an inverse air-gap function $\left(g^{-1}\right)$ by the rotor flux path and the WF $\left(\mathbf{N}_{\mathbf{A}}\right)$ by the stator flux path. The flux path due to the magnetic saliency of the rotor is calculated by the equivalent permeance value, and the flux path due to the stator slots can compute as in Fig.4.

$$
\begin{aligned}
g_{s}(\theta) & = \begin{cases}g_{0}+\frac{\pi}{2} R \theta & \text { for } 0 \leq R \theta \leq \mathrm{k}_{0} \\
g_{0}+\frac{\pi}{2} R \theta+\gamma\left(R \theta-\mathrm{k}_{0}\right) & \text { for } \mathrm{k}_{0} \leq R \theta \leq \tau / 2\end{cases} \\
\gamma & \left.=(\pi / 2)-\arctan \left(\mathrm{k}_{1} /\left(\mathrm{w}_{1}-\mathrm{w}_{0}\right) / 2\right)\right)
\end{aligned}
$$

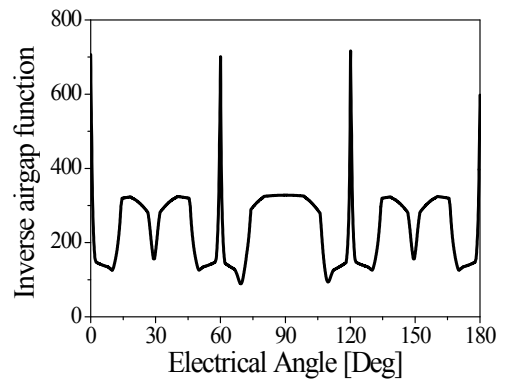

Fig. 5. Distribution of $g^{-1}$ in the air-gap.

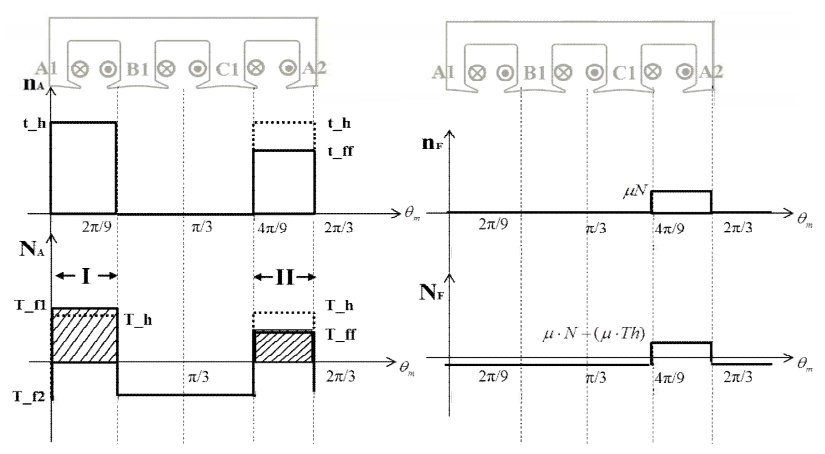

Fig. 6. TF $\left(\mathbf{n}_{\mathbf{A}}\right)$ and $\mathrm{WF}\left(\mathbf{N}_{\mathbf{A}}\right)$ under the STF condition.

As shown in (11), $g^{-1}$ can be obtained from the modeling of the flux path in the air-gap. Finally, the air-gap flux density of the $A$-phase can be calculated as a product of $g^{-1}$ and $\mathbf{N}_{\mathbf{A}}$, as shown in (12). Therefore, the inverse-gap function is calculated as Fig. 5.

$$
\begin{aligned}
& g^{-1}\left(\theta_{m}-\varphi\right)=\frac{1}{g_{s}\left(\theta_{m}\right)+g_{r}\left(\theta_{m}-\varphi\right)} \\
& B_{A}\left(\theta_{m}\right)=\mu_{0} g^{-1}\left(\theta_{m}\right) N_{A}\left(\theta_{m}\right) i_{A}
\end{aligned}
$$

where, $g_{s}\left(\theta_{m}\right)$ is the flux path due to the stator slots; and $g_{r}\left(\theta_{m}\right.$ $-\varphi)$ is the air-gap function.

Fig. 6 shows the WF and TF under healthy conditions and under the STF condition. $\mathbf{n}_{\mathbf{F}}$ and $\mathbf{N}_{\mathbf{F}}$ are defined as TF and WF under the STF condition. The other functions are defined as:

$t_{-} h=\frac{N}{3}:$ TF under healthy state

$t_{-} f f=\frac{N}{3}(1-\mu):$ TF under ITF state

$T_{-} h=\frac{N}{3}-\frac{N}{6}$ : WF under healthy state

$T_{-} f 1=\frac{N}{3}-\frac{N}{6}(1-\mu):$ WF of other $A$-phase under ITF state

$T_{-} f 2=-\frac{N}{6}(1-\mu):$ WF under ITF state

$T_{-} f f=\frac{N}{3}-\frac{N}{6}(1-\mu)-\mu N:$ WF of fault $A-$ phase under ITF state

Through $\mathbf{n}_{\mathbf{F}}$ and $\mathbf{N}_{\mathbf{F}}$, the inductance under the STF condition can be obtained. In addition, these functions can present the 


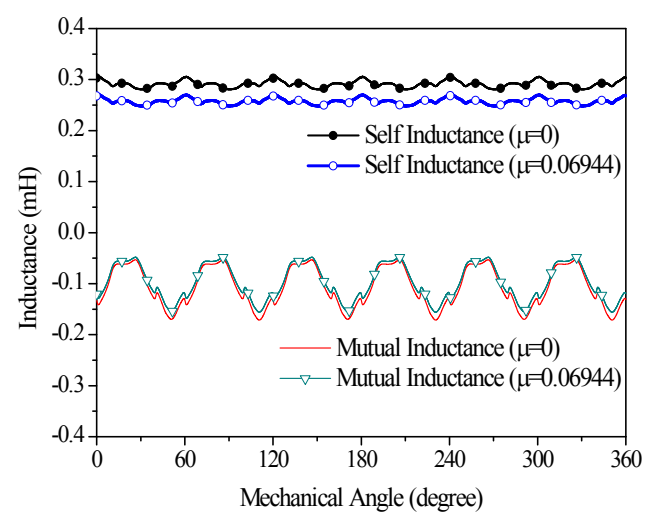

Fig. 7. Inductance variation by STF.

teeth magnetic density with faults by calculating the I-region in the faulty phase and the II-region in the faulty phase. The fault has not yet spread to I-region but it has spread to II-region. Therefore, each part of the inductance and magnetic flux density can be calculated from (13). Finally, the distributed flux density can be calculated using the proposed equation.

$$
\begin{aligned}
B_{\text {region_I }} & =\frac{\left\{L_{\text {region_I }} \cdot i_{a} /\left(N_{\text {per_teeth }}\right)\right\}+\varphi_{P M}}{A_{\text {Teeth }}} \\
B_{\text {region_II }} & =\frac{\left\{L_{\text {region_II }} \cdot i_{a} /\left(N_{\text {per_teeth }}-\mu N_{\text {per_phase }}\right)\right\}+\varphi_{P M}}{A_{\text {Teeth }}}
\end{aligned}
$$

\section{SIMULATION RESULTS}

Many electrical devices contain non-linear magnetic systems. In particular, in IPM motors, the magnetic non-linearity leads to significant variations in the flux-related machine parameters, specifically, the self-magnetization and mutual inductances. Although the proposed analytical method does not consider non-linearity, it can approximate the variation pattern of the distributed magnetic characteristics under the STF condition. The stator inductance in the healthy state is $0.3(\mathrm{mH})$. However, it decreases to $0.25(\mathrm{mH})$ when the fault fraction is $8.33 \%$. It has been confirmed that the flux and self-inductance decrease because of a shorted coil, based on the inductance profile shown in Fig. 7.

Fig. 7 shows the variations of the radial flux density at the stator teeth based on the distributed magnetic characteristic analysis. The flux density at the stator teeth changes as the input current varies and the fault fraction changes. Fig. 8 (a) shows the calculation results for the faulty phase using the proposed model. Fig. 8 (b) considers the effect of the B-H curve of the core using the FEM. The results obtained with the proposed analytical model are slightly different than those obtained with the FEM, because it cannot consider the non-linear characteristics up to the point of saturation. The distributed flux density field of the FEM is presented in Fig. 9. The proposed model generates a circulating current in the opposite direction to that of the magnetic field. Therefore, as the fault fraction increases over time, a reverse magnetic field

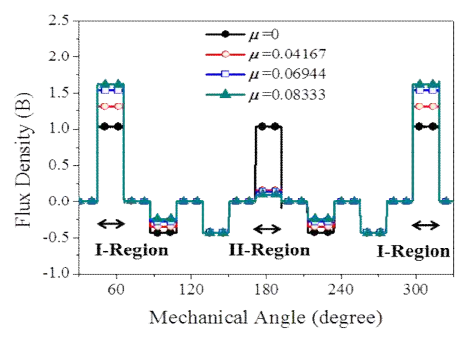

Mechanical Angle (degree)

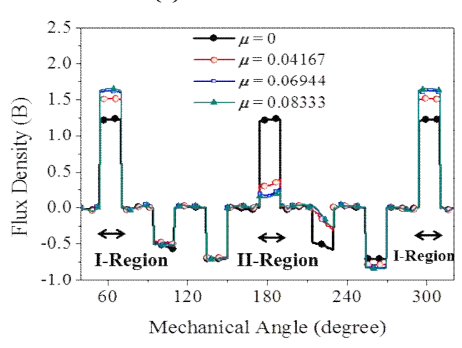

(b) FEM result

Fig. 8. Variation of flux density.

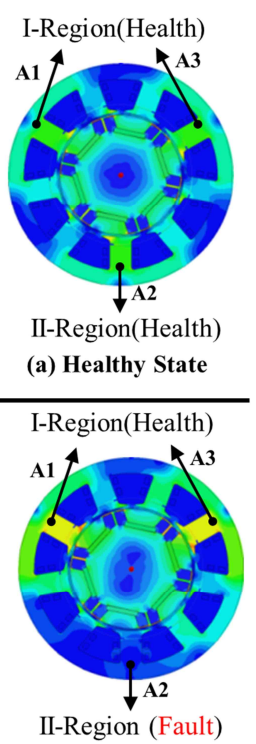

(b) Faulty State

Fig. 9. Distributed flux density.

is generated in the teeth with the shorted turn and magnetic saturation occurs in the other teeth. When a large number of turns are involved in an STF, the additional flux strongly distorts the distribution of the magnetic flux in the air gap.

\section{EXPERIMENTAL RESULTS}

The distributed magnetic characteristics under the STF condition can be monitored because the circulating current generates a distinct unbalanced magnetic field. To validate the distributed magnetic characteristics, an experiment has been performed using a detection coil, which generates an induced voltage based on the time-varying magnetic field. In addition, as shown in Fig. 10, this experiment applied a LPF (low-pass filter) for harmonic filtering in the switching of the inverter. Using (15), the linkage flux can be calculated using the output 


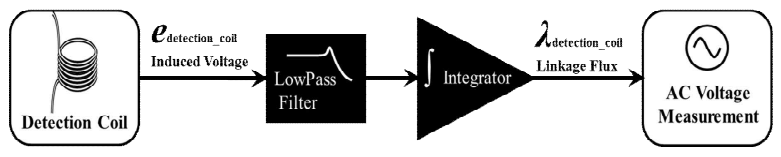

Fig. 10. Experiment process for DMC detection.

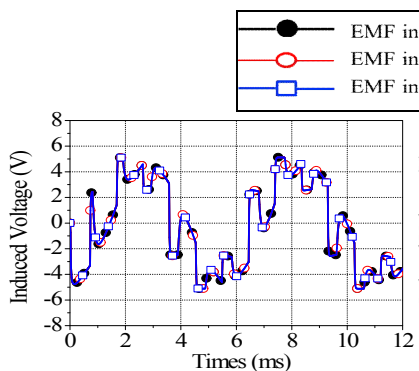

(a) EMF by analytical model $(\boldsymbol{\mu}=0 \%)$

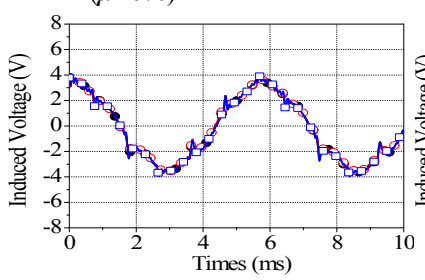

(c) EMF by experiment $(\boldsymbol{\mu}=0 \%)$

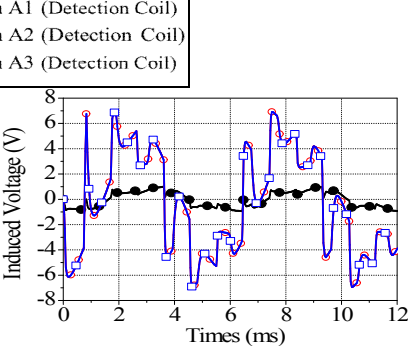

(b) EMF by analytical model $(\boldsymbol{\mu}=12.5 \%)$

(d) EMF by experiment $(\mu=12.5 \%)$

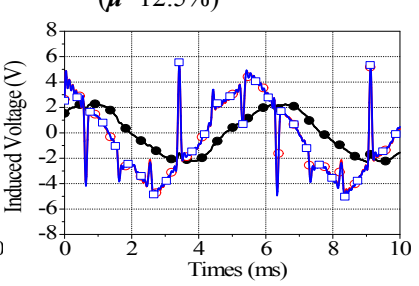

Fig. 11. Induced voltage in the detection coil.

of the integrator. $K_{\text {Integrator }}$ and $K_{L P F}$ are the gains of the integrator and the LPF, respectively. Fig. 11 and Fig. 12 show the EMF voltage, which is the voltage induced by the rotating magnetic field, and the linkage flux, which is calculated using (14), respectively. In Figs. 11 (a) and (b), the analytical results confirm the EMF variations caused by a STF. Furthermore, in Figs. 11 (c) and (d), the experimental results confirm the EMF variations caused by a STF. From the experimental results shown in Fig. 12 (d), it can be seen that the value of the linkage flux is one thousand times higher than the simulation value for the gain value of the integrator. An experimental motor was designed for testing STFs and for applying detection coils, as shown in Fig. 13. The detection coils are wound around each tooth, and each coil carries a phase winding for the detection of the distributed magnetic characteristics.

$$
\lambda=K_{\text {Integrator }} \cdot \int\left(K_{L P F}\right) \cdot\left(e_{\text {detection_coil }}\right) d t
$$

\section{CONCLUSIONS}

This paper proposed an analytical model for calculating the distributed magnetic characteristic with STFs using the WFT. Regarding the STFs, it is possible to determine which one has the most problematic characteristics. Proposed analytical modeling was developed on the basis of the analysis of STFs using mechanical equations. Thus the distributed magnetic field were analyzed using a WFT that uses these analytical functions. This has been verified using both FEM-simulation

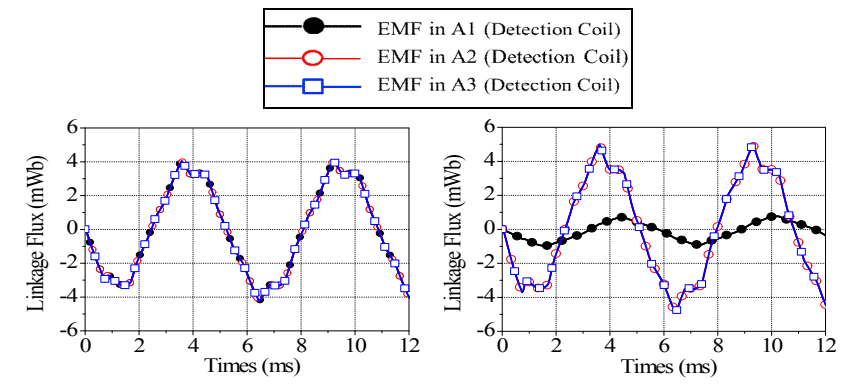

(a)Linkage flux by analytical model (b)Linkage flux by analytical model

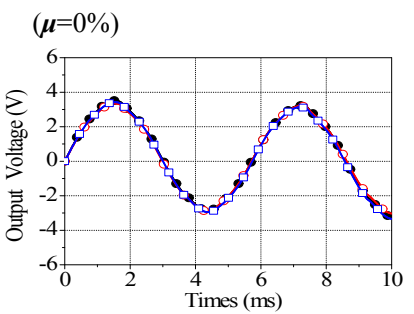

(c)Linkage flux by experiment $(\boldsymbol{\mu}=0 \%)$

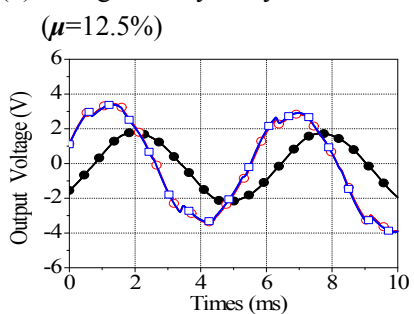

(d)Linkage flux by experiment $(\boldsymbol{\mu}=12.5 \%)$
Fig. 12. Output value in the detection coil.

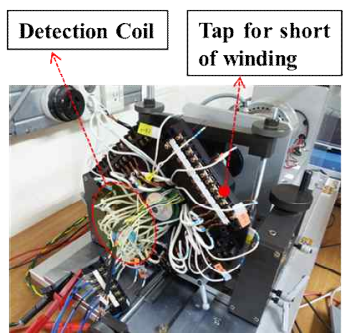

Fig. 13. Test motor.

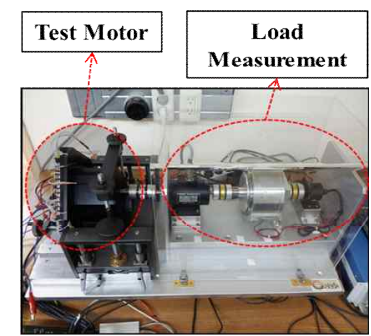

Fig. 14. Test equipment and motor.

and experimental results.

The proposed analytical modeling has good agreement although it uses a simple calculation process. It can be used for fault-tolerant operations by detecting the STF at the initial stage and small fault fraction.

\section{ACKNOWLEDGMENT}

This work was supported by the Energy Efficiency \& Resources of the Korea Institute of Energy Technology Evaluation and Planning (KETEP) grant funded by the Korea government Ministry of Science, ICT \& Future Planning (No. 2012T100201723) and the MSIP (Ministry of Science, ICT \& Future Planning), Korea, under the C-ITRC (Convergence Information Technology Research Center) support program (NIPA-2013-H0401-13-1008) supervised by the NIPA (National IT Industry Promotion Agency). 


\section{REFERENCES}

[1] J. Hur, "Characteristic analysis of interior permanent magnet synchronous motor in electro-hydraulic power steering system," IEEE Trans. Ind. Electron., Vol. 55, No. 6, pp. 2316-2322, Jun. 2008.

[2] Y. K. Lee and T. G. Habetler, "A phase variable simulation model for interior PM synchronous motor drives with stator turn fault," EPE-PEMC'06. 12th IEEE, pp. 1074-1079, 2006

[3] T. Kim, H.-W. Lee, and S. Kwak, "The internal fault analysis of brushless DC motors based on the winding function theory," IEEE Trans. Mag., Vol. 45, No. 5, pp. 2090-2096, May 2009.

[4] J. A. Farooq, A. Djerdir, and A. Miraoui, "Modelling and simulation of stator winding inter-turn faults in permanent magnet synchronous motors," COMPEL: The International Journal for Computation and Mathematics in Electrical and Electronic Eng. , Vol. 27, No. 4, pp. 887-896, 2008.

[5] O. A. Mohammed, S. Liu, and Z. Liu, "FE-based physical phase variable model of PM synchronous machines under stator winding short circuit faults," IET Sci. Meas. Technol., Vol. 1, No. 1, pp. 12-16, Jan. 2007.

[6] O. A. Mohammed, Z. Liu, S. Liu, and N. Y. Abed, "Internal short circuit fault diagnosis for PM machines using FE-based phase variable model and wavelets analysis," IEEE Trans. Mag., Vol. 43, No. 4, pp. 1729-1732, Apr. 2007.

[7] B. Vaseghi, N. Takorabet, and F. Meibody-Tabar, "Fault analysis and parameter identification of permanent-magnet motors by the finite-element method," IEEE Trans. Mag., Vol. 45, No. 9, pp. 3290-3295, Sep. 2009.

[8] L. Romeral, J. C. Urresty, J. R. Riba Ruiz, and A. Garcia Espinosa, "Modeling of surface-mounted permanent magnet synchronous motors with stator winding inter-turn faults," IEEE Trans. Ind. Electron., Vol. 58, No. 5, pp. 1576-1585, May 2011.

[9] T. Lubin, T. Hamiti, H. Razik, and A. Rezzoug, "Compairson between finite-element analysis and widing function theory for inductances and torque calculation of a synchronous reluctance machine," IEEE Trans Mag., Vol. 43, No. 8, pp. 3406-3410, Aug. 2007.

[10] Q. Wu and S. Nandi, "Fast single-turn sensitive stator inter-turn fault detection of induction machines based on positive and negative sequence third harmonic components of line currents," Industry Applications Society Annual Meeting, 2008. IAS '08. IEEE, pp. 1-8, 2008.

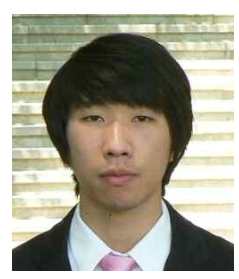

Kyung-Tae Kim was born in Busan, Korea. He received his B.S. and M.S. in Electrical Engineering from the University of Ulsan, Ulsan, Korea, in 2010 and 2012, respectively. Since 2012, he has been working towards his Ph.D. at the University of Ulsan. His current research interests include motor design and motor diagnosis.

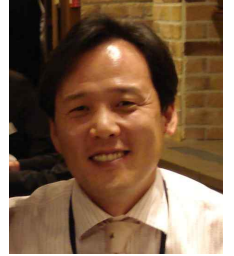

Jin Hur (''93-M'98-SM'03) received his $\mathrm{Ph.D.}$ in Electrical Engineering from Hanyang University, Seoul, Korea, in 1999. From 1999 to 2000, he was with the Department of Electric Engineering, Texas A\&M University, College Station, TX, as a Postdoctoral Research Associate. From 2000 to 2001, he was a Research Professor of Electrical Engineering for BK21 projects at Hanyang University. From 2002 to 2007, he was a Director of Intelligent Mechatronics Research Center, Korea Electronics Technology Institute (KETI), Puchon, Korea, where he worked on the development of special electric machines and systems. Since 2008, he has been an Associate Professor, School of Electric Engineering, University of Ulsan, Ulsan, Korea. He is the author of over 140 publications on electric machine design, analysis and control, and power electronics. He has 1 granted pending US patent and 20 granted pending Korean patents. His current research interests include high-performance electrical machines, modeling, drives, new concept actuators for special purposes and numerical analysis of electromagnetic fields. Dr. Hur is working as an Associate Editor for IEEE Transaction on Power. He is also an IEEE Senior Member and a Technical Program Chair of the IEEE VPPC2012 Conference.

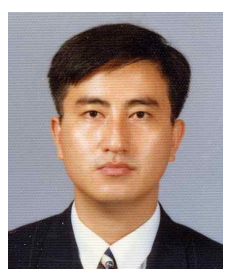

Byeong-Woo Kim received his B.S. and M.S. in Mechanical Engineering from Hanyang University, Seoul, Korea, in 1987 and 1990, respectively and his Ph.D. in Precision Mechanical Engineering from Hanyang University, in 2002. From 1989 to 1990, he was an Invited Researcher at the Precision Measurement Group, Japan Kosaka Research Institute (JKRI), Saitama, Japan. From 1990 to 1994, he was a Junior Researcher at the Sensor Development Group, CAS Cooperation, Seoul, Korea. From 1994 to 2006, he was a Principal Researcher at the Electrical \& Electronics Group, Korea Automotive Technology Institute (KATECH), Chunan, Korea. In 2006 Dr. Kim joined the School of Electrical, Electronics and Information Systems Engineering, Ulsan University, Ulsan, Korea, as an Associate Professor. His current research interests include electrical and electronics systems for automotive applications, and control systems for hybrid, fuel cell and electric vehicle systems. 